\title{
A Review and Reappraisal of Task Guidance: Aiding Workers in Procedure Following
}

\author{
Jennifer Ockerman and Amy Pritchett \\ School of Industrial and Systems Engineering \\ Georgia Tech
}

\begin{abstract}
Procedures can greatly benefit workers at tasks such as inspection, maintenance, and assembly. Procedures may serve as a guideline for expert workers or they may provide a list of directives to be followed exactly; either way, procedures serve to structure a task, to aid worker memory, and to guarantee consistency and safety. Light, inexpensive electronics may allow for the development of task guidance systems to further help workers by presenting procedures and associated information about the task. This article reviews the current state of knowledge about the development of task guidance systems, and highlights their potential value in a variety of domains. First, the characteristics of procedural tasks are discussed, as a basis for a discussion on the benefits of procedure following that task guidance systems can support, and potential problems in procedure following that task guidance systems can mitigate. Then, current research results in task guidance systems are summarized. Finally, a discussion is given on the contextual information that a task guidance system may need to provide.
\end{abstract}

\section{INTRODUCTION}

Commensurate with the development of increasingly small, light, and mobile electronics is the need to understand how this technology may be used to help workers in industrial settings. Systems such as personal data assistants and wearable computers have recently (and quickly) achieved the critical set of qualities that make them capable of assisting a variety of tasks. One potentially valuable function may be to guide workers through proceduralized tasks, such as inspection, maintenance, and assembly. Although this type of task is pervasive to almost every industry, most of the attention has been paid to designing the underlying procedure (rather than designing task-aiding technology) or to automating the worker out of the task entirely; assisting the user through a supplementary task guidance system remains a comparatively novel course of action.

This article proposes the term task guidance systems for electronic worker-aids, which, without any sensing of the environment at hand, can serve as a reference and guide to the worker. New technological capabilities make such systems practicable. Inspection and assembly tasks often require hands-free operation; task guidance systems can now talk and

Requests for reprints should be sent to Amy Pritchett, School of Industrial and Systems Engineering, Georgia Tech, Atlanta, GA 30332-0205. E-mail: jojo / amy.pritchett@isye.gatech.edu 
listen to the worker. These tasks often require the worker to be mobile during a task; task guidance systems can now be carried around —or worn — with relative ease. These tasks require the worker to traverse many-sometimes hundreds — of steps; task guidance systems can now store and display them in a consistent format and in the right order. Finally, these systems are now sufficiently inexpensive that their widespread use is conceivable in communities such as mechanics and factory workers where no such electronic assistance has been provided before, if they are designed well enough to have a clear, demonstrated benefit to workers.

However, for task guidance systems to be beneficial, they must be developed with the worker's task in mind. A number of problems may occur, ranging from the system interfering with the primary task and being ignored, to the worker overrelying on the system in conditions where its underlying procedure is inappropriate or incorrect. Conversely, on a positive note, if the task guidance system is viewed as more than just an electronic version of a paper checklist, it may use its display flexibility and memory store to provide a powerful assistant to a worker.

This article examines task guidance design by starting with a systematic discussion of proceduralized tasks' characteristics and worker capabilities. This discussion will serve as a basis for understanding how task guidance systems may support the worker. Then, studies of task guidance systems are discussed and contrasted with the more established research on automation design. The specific information about context that task guidance systems may need to provide is detailed, and finally the issues remaining with the development of task guidance systems and with the use of procedures are discussed.

\section{PROCEDURES}

\subsection{Potential Benefits of Procedure Following}

To understand how task guidance systems may aid a worker at procedural tasks, such as inspection, maintenance, and assembly, it is essential to understand the nature of these tasks. Imagine, for example, standing in front of a large, air transport aircraft newly deposited in your maintenance hangar, knowing that you and your team have been tasked with performing a major airworthiness inspection. Although a trained, skilled mechanic, you may never before have worked on this type of aircraft or on a specific subsystem that this specific aircraft has installed. Without a procedure to follow, the task would lack structure; team members would require constant coordination; some aircraft parts might miss being inspected; and other aircraft parts might be handled in an order that is inefficient, such as reinstalling one part before a part underneath it has been inspected.

In domains such as inspection, maintenance, and assembly, the development of a procedure is a vital preliminary step toward completing a task safely and consistently. Procedures bear a sizeable resemblance to plans, except for the connotation of a procedure being mandated by an authority. Even in day-to-day life, planning a day's events in a busy family may result in checklists for the kids, shopping lists for one parent, and a timetable of pick-ups and dropoffs for the other parent, all written down and posted like procedures. In more industrial examples, anesthesiologists report creating "points for consideration" before a surgery as quick reminders of vital signs to check and actions to take under a variety of conceivable conditions (Xiao, Milgram, \& Doyle, 1997). Likewise, plans for military air missions may be detailed and rehearsed to a level of detail found in civil aviation, with con- 
tingencies for likely eventualities. Carrying this aviation concept further, onboard planning tools have been proposed for helping pilots prepare on-the-fly procedures in response to situations where normal procedures do not apply, which the pilots can then follow throughout the remainder of the flight as they would a standard operating procedure (Chen \& Pritchett, 2000).

When a task includes many steps, its sheer magnitude warrants presenting the task with a beginning, an end, and a manageable sequence of steps in between. The safety and efficiency of the procedure can be established beforehand, when the procedure is designed. There are several different ways in which a procedure may structure a task, each with their comparative benefits in terms of efficiency and comprehensibility. For example, in inspecting an aircraft it may be more efficient to do a "structural search," in which the order of inspection follows the physical structure of the aircraft, walking around it and examining each component in turn, be it airframe, hydraulic, or electrical in nature. In contrast, to help diagnose and trace out pilot-reported problems with an aircraft, a mechanic may prefer to perform a "functional" search, in which each type of component is checked together, such as examining together all electrical components whether on the nose, the tail, or the wing tips.

Ultimately, a procedure takes a large, complex task, capable of being approached in many different ways, and presents it as a sequence of manageable, communicable steps. For some tasks, the specific ordinality of the steps may be critical, such as many assembly and maintenance tasks in which a component must be assembled with its parts in an exact order and relative position, especially when some steps are irreversible. In even more demanding cases, the sequence may have an imbedded temporal component. For example, the specific order of actions to restart the engine following an engine failure in a Cessna 172 is very important-Carb heat ON, Fuel Selector Valve BOTH, Mixture RICH, Magnetos LEFT-RIGHT-BOTH, Master ON, Primer IN and LOCKED (Cessna, 1975). Not only does this list contain the actions that will respond to the most likely sources of the problem, but it also proscribes them in an order that puts first those requiring a still-warm engine and the most time to take effect. In inspection, maintenance, and assembly tasks, temporal constraints may arise, for example, when mixing chemicals or when dealing with paints and glues that can only be applied within a certain time following exposure to air.

Although a procedure typically issues a linear sequence of steps to the worker, the underlying dynamics of the task may be far more intricate. For example, aspects of the environment may require a change in the sequence; the procedure may know the relevant aspects of the environment and their effect on the sequence, but only the worker can assess the environment. In that case, the worker is prompted not only on what to do, but also on what to look for; conditional statements may be imbedded in the procedure, such as "If the aircraft has a Mode $S$ transponder installed, then follow the steps listed on page 47..." Such statements may require the worker to fork to a different procedure, or may represent movement within a hierarchy of high-level and low-level procedures. The resultant impact on the worker, however, is that she or he is prompted on a sequential set of steps to complete, be they actions or assessments of the environment, without requiring him or her necessarily to track the various hierarchies and conditionals imbedded within the procedure.

This "prompter" role highlights the assistance a procedure can provide to the worker. By presenting the worker with a list of easily understood steps, they provide a memory-assistant that mandates a consistency and safety level that the worker might otherwise "forget" to achieve. Several reasons for this memory aid exist. Most obviously, the number of steps may be too large for any human to accurately remember. In operational settings, it is also 
not uncommon for workers to be asked to perform tasks they have never executed before, such as inspecting a different type of aircraft or transitioning to a different assembly line in the factory; in these cases, she or he has no past experience with the exact procedure to remember, even though she or he is capable of executing it when given. Even when the list of steps is well known, memory lapses may occur. Of particular interest to procedure following is the effect of interruptions during sequential tasks; several studies have shown that interruptions can cause the worker to skip steps or forget their place in the procedure (Latorella, 1996; Linde \& Goguen, 1987).

Although giving the worker a procedure may help with memory, it is important to note what the procedure does-and does not-know. Implicit in a procedure is predetermined knowledge about the task at hand; this information may include directive information specifying immediate actions, feed-forward information highlighting upcoming steps, and feedback information specifying checks and confirmations to make on just-completed steps (Drury \& Prabhu, 1996; Patel, Drury, \& Lofgren, 1994). What is missing, however, is any current knowledge of the environment (environment information) and, as a specific subset of knowledge of the environment, any assessment of the state of the system being operated on (operand information). This real-time assessment of the environment and operand remains the responsibility of the worker at all stages-initiating a step, completing a step, and examining feedback from the environment to confirm the step has been completed properly.

Just as a procedure does not intrinsically have knowledge of the environment, so does a procedure not necessarily remove the need for an expert to complete the task. Instead, the expertise of the worker is represented not by an ability to plan a set of actions to follow, but by an ability to translate what the written words say into comprehension of what they mean, to understand the steps, to relate them to the environment, and to execute the actions that are required. For example, an aircraft maintenance procedure may list as a single step, "If the valve appears to be damaged, either replace with a factory part or machine a replacement valve according to the blueprint. Confirm that the replacement part conforms to the specification with a tolerance no greater than 7/1000 of an inch once installed"; the craftsmanship required is referred to only casually by the procedure. Likewise, many cockpit emergency procedures end with the step "Land as soon as possible," a command requiring considerable manual control skills, quick thinking, and judgement for reliable execution.

In short, although procedures may need to be designed by experts, they may also require experts (or at least skilled workers) to execute them. For this reason, a worker can commonly be judged by his or her ability to execute a procedure correctly, with training explaining how to complete each step, and testing requiring demonstration of such. The consistent explicit reference to written procedures is considered in many domains a trademark of professionalism and precision, such as piloting aircraft. For example, one pilot training manual gave this advice for the commercial pilot flight test: "You will use the manufacturer's recommended procedure or you will have your own ... Of course, you [sic] have used a thorough [checklist] for every flight" (Kershner, 1976, p. 236).

The efficacy of a procedure may be measured by three attributes: the comprehensiveness of the procedure, indicating the range of situations, environments, and operands the procedure is suitable for; the detail of the procedure, indicating the degree to which each step within the procedure specifies the exact actions the user should follow; and the accuracy of the procedure, indicating the extent to which the steps will produce the desired results. It is easy for a procedure to have a high score on any two of these attributes and still not be sufficient for the task. For example, a single-step procedure, "Inspect the aircraft," 
is both accurate and comprehensive, albeit lacking in detail; likewise, an engine-expert may be able to make an accurate and detailed procedure for the engine, but which is not comprehensive for the remainder of the aircraft. The challenge posed to the procedure developer is to establish a procedure whose attribute measures attain the levels most suited to the worker's needs.

Different amounts of comprehensiveness and detail are suited to different procedure-following strategies. For example, procedures for assembly tasks tend to be "do-lists," which can be completely comprehensive because each action usually is known in advance. In comparison, procedures for inspection tasks may never be able to span the complete range of failures and faults that may be present; therefore, they are typically "check-lists," which provide a sample of the most important things to check, with the expectation that the worker will also be on guard for unexpected faults not included in the procedure.

When the procedure is comprehensive and detailed, the worker's task is to follow the procedure exactly, without expecting the worker to conceptualize the task outside of the individual steps. For example, although specific thought may have gone into designing the Cessna engine restart procedure mentioned earlier, the pilot can just follow the procedure exactly, without needing to conceptually compare the timing and efficacy of various engine controls. In such conditions, the worker can free up his or her time and attention for other tasks such as controlling the aircraft, planning a trajectory to a safe forced-landing, and so forth.

In other conditions, procedures may not need to be fully comprehensive and detailed. For example, an investigation of the use of nuclear power plants (Roth, 1997) found that workers did not simply follow the procedures they were given, but used higher level cognitive skills to assess the situation, decide on the appropriateness of the procedure for the situation, and prepare for future steps in the procedure. Interviews of operators of nuclear and process control plants noted a preference for procedures to serve as guidance, presented in a concise form that describes tasks only in terms of important details and placing "more reliance on the personal initiative and experience of the operator" (Seminara, Gonzalez, \& Parsons, 1976, as quoted in Marsden, 1996, p. 111; Zach, 1980). Likewise, the use of different "techniques" for following procedures has been noted by Degani and Wiener $(1994,1997)$ with airline pilots in employing the same cockpit checklists and by Pearl and Drury (1995) with airline mechanics and maintenance procedures.

The type of strategy to use is largely stipulated by the worker's knowledge of the task, and the flexibility that can be shown in completing it. The "lock-step" strategy requires a comprehensive, detailed, and accurate procedure, and is typically suited for conditions in which the worker is unfamiliar with the task and the procedure can provide acceptably safe, reliable guidance. It is also needed in conditions in which procedures guide interactions between multiple players, such as air traffic control. In comparison, the "guidance" strategy is typically suited for situations in which the worker is familiar with the task, and hence lower procedure comprehensiveness and detail is acceptable. It also requires that the task be amenable to variations in worker behavior-although many tasks can benefit when the worker has the flexibility to adjust their actions to that most appropriate to the immediate context, some tasks may have a single course of action that can successfully lead to completion.

Just as the worker may approach the task with different strategies, so too may he or she approach the procedure differently. With greater knowledge of the procedure, the worker may prefer to use an anticipatory strategy in applying the procedure. In doing so, the worker 
can think ahead and accommodate their current actions for upcoming steps; for example, a mechanic may elect to not stow some tools after performing one step based on the knowledge that they will be needed again soon. Studies in three different domains have illustrated performance advantages in using an anticipatory strategy: productivity gains with factory workers (Grosjean, 1995); preparedness of potential off-nominal conditions with anesthesiologists (Xiao et al., 1997); and timeliness and efficiency in nuclear power plant operators (Roth, 1997).

The ability to execute an anticipatory strategy may come from several sources. In the studies just described, the anticipatory strategy was enabled by expertise; Grosjean (1995) also noted that factory workers were better able to apply an anticipatory strategy when the environment (in this case, the machines the workers operated) followed a more regular pattern. An anticipatory strategy may also be facilitated by an expectation of probable events such as the anesthesiologist example provided earlier. It has been found that providing feedforward information in training or daily job interviews (Drury \& Prabhu, 1996; Patel, Drury, \& Lofgren, 1994) can improve performance in inspection tasks (Sheehan \& Drury, 1971). For example, in this last study, electronics inspectors were provided with feedforward information about the likely faults to be found in components based on knowledge of the manufacturing process.

In summary, procedures have the potential to greatly assist workers, assuming they are accurate. The comprehensiveness and detail of the procedure can be tailored to the type of strategy the worker will use to conduct the task, given the worker's training and expertise and the demands of the task environment. The inclusion of feed-forward information and worker familiarity with a procedure can also encourage an anticipatory approach to the task.

\subsection{Potential Problems With Procedure Following}

Unfortunately, the potential benefits of procedures are not always fully realized. When the procedure provides an accurate task structure and the worker does not follow it-intentionally or unintentionally-issues with procedural noncompliance (also called nonconformance or underreliance) may arise. Depending on the task, the range of actions outside of the procedure that result in satisfactory performance, and the domain expertise of the worker, noncompliance may not be an issue. However, in domains with little leeway for nonprocedural actions, noncompliance can dramatically impact safety and efficiency; for example, improper (or lack of) procedure use has been cited in several aircraft accidents and incidents (Mosier, Palmer, \& Degani, 1992; Palmer \& Degani, 1991; Pearl \& Drury, 1995).

Noncompliance may stem from several sources. Unintentional noncompliance may arise due to memory lapses and misinterpretation of a step. Intentional noncompliance, on the other hand, can arise when the worker perceives more drawbacks to following a procedure than benefits; unfortunately, many procedures currently being used meet this description (Drury \& Prabhu, 1996; Drury \& Sarac, 1997; McCarthy, Wright, Monk, \& Wattts, 1998; Patel, Pearl, Koli, \& Drury, 1994; Thomas, 1995).

A worker may want to use a procedure in theory, but find it overly burdensome to do so in actuality. Generally, the worker must carry a physical representation of the procedure with him or her, frequently divert attention to reading the procedure (if written down), and apply shallow reading skills (Hutchins, 1995, p. 295). The manner of presenting the procedure to the worker may make these processes difficult if its physical representation is too 
heavy; too delicate, such as paper checklists needing to be used outside in the rain; immobile, such as work-station presentations of procedures to mobile workers; or too difficult to read, whether through poor formatting or poor choice of words (Pearl \& Drury, 1995).

Conversely, even when procedures are easy to access, workers may see insufficient benefit to follow them. Although the individual steps may be easy to understand, the entire sequence of steps may be different from what the worker is accustomed to or trained on; it may be seen as inefficient or overly difficult; or it may be perceived as being wrong (Pearl \& Drury, 1995). Organizational culture can play a significant role in the perceived benefit of following a procedure (Marsden, 1996). Although in some domains procedure following is held as a mark of precision and professionalism, in other domains the organization may condone, even reward, procedural noncompliance explicitly or implicitly. At the extreme, countercultures may be found amongst workers, viewing procedures as "a system of work control designed essentially to protect the company in the event of an accident" (Marsden, 1996, p. 111).

In summarizing the previous section on the benefits of procedures, the assumption was stated that the procedure was accurate. Unfortunately, in too many instances, procedures have been found to be faulty. For example, Marsden (1996, p. 99) summarized two studies of procedure following in the nuclear industry. The first (INPO, 1986) reported that, of the $48 \%$ of incidents initially attributed to "failures of the human factor," almost $65 \%$ involved a procedural deficiency. The second reviewed almost 700 incidents and concluded that faults in procedures were implicated in 69\% (Goodman \& DiPalo, 1991).

Both the overall procedure and each of its component steps are intended to implicitly assure the worker that the given set of actions will meet a specific intention within a range of environmental configurations and worker capabilities (boundary conditions). When the worker has a different intention than the procedure, when the environment or worker capabilities do not fall within the range covered by the procedure, or the set of actions is outright wrong, the procedure cannot be relied on. Unfortunately, studies have found that workers often cannot judge the appropriateness of the procedure (e.g., Schutte \& Trujillo, 1996).

Designing a procedure a priori to simultaneously be comprehensive, detailed, and accurate can be quite difficult. In attempting to be comprehensive, a procedure must meet a variety of intentions and span a wide range of boundary conditions to maintain its accuracy; a detailed procedure faces similar problems in being accurate in details, which may be contingent on environmental factors and therefore susceptible to exceeding boundary conditions. Within these bounds, if several procedures are possible, it is additionally desirable to select the one most harmonious with the training and work practices of the worker (Vicente, 1999).

These difficulties then continue throughout the life of a procedure-just as facilities and training may be updated, so may a procedure need to be changed in response to changing conditions. For example, following the failure of a Titan IVB launch of a missile warning satellite due to a thermal wrap around a connector that prevented proper disconnection of the first and second stages of the launch vehicle, an investigation found that "personnel apparently did their jobs as indicated by instructions, but the 21-year old 'detailed operating procedures' were flawed ... the [operating procedure] application of the thermal wrap was consistent with the engineering drawing of the vehicle assembly ... dating back to 1978 [which] omitted unique requirements for the [new] separation function" (Covault, 1999, p. 34).

If the potential for procedure failures exists, two main responses may be made to guard against the worker blindly enacting incorrect procedural directives. The first may be to re- 
duce the detail of the procedure. This may force the worker to use a strategy in following the procedure that actively requires the worker to draw on his or her expertise and, hopefully, act as an error-checking mechanism. This typically requires highly trained workers, and may not provide all available guidance in common, nominal conditions - or in emergency conditions requiring specific immediate responses.

The second preventive measure may be to make clear to the worker the intention and boundary conditions of the procedure, as well as its conceptual framework. This approach thereby provides the worker with the information required to asses the appropriateness of the procedure for the task and conditions at hand; if the worker has sufficient expertise, then he or she may also be able to judge the accuracy of the procedure. This approach requires identifying the salient information about the procedure to present and methods to display it-the subject of a later section.

\section{TASK GUIDANCE SYSTEMS}

\subsection{Physical Instantiations of Procedures}

Although Webster (1980) defines a procedure in conceptual terms- "a course or mode of action; a step taken"- the instantiation of a procedure in a physical form is so commonplace that the term procedure typically conjures the vision of a paper checklist—art and artifact are intrinsically linked in practice. Several factors necessitate the written form of the procedure: a need to communicate and share it; an inability to remember a long procedure; and a desire to confirm how to perform important actions and verify that they have been completed correctly.

Physical instantiations of procedures are usually intended to be easy to use, acting as a one-way memory cue to the worker that requires nothing in return beyond the flip of a page or a check beside each step as it is completed. Interaction with a checklist, for example, typically requires occasional attention and application of shallow reading skills to translate external written words into an internal representation of what the writing says (Hutchins, 1995, p. 298).

Although written checklists can be easy to use and meet several basic needs, they are not perfect. Even when the underlying procedure is correct, studies of paper checklists have identified several types of errors in their use: losing track of the "current" step, and therefore skipping steps; skipping items due to interruptions and distractions; intentionally skipping an item and forgetting to return to it; and stating that an item has been completed when it has not (Palmer \& Degani, 1991).

The use of written checklists has also faced more mundane difficulties. When the procedure is long and complex, the written checklist may be large, bulky, heavy, and distributed over several volumes. When procedures must be updated, both providing new documentation and rigorously eliminating old documentation can present a fair logistical problem. Paper checklists may be difficult to hold in many environments defined by attributes such as cold, hot, wet, or windy, and difficult to access in positions where the worker needs their hands free or the space around them clear. Finally, when the procedures require the worker to jump forward and backward in the list of steps, navigation through written checklists may not be easy, especially if steps to be performed sequentially are not colocated physically in the same list. 


\subsection{Task Guidance Systems: Definition}

Task guidance systems - a specific term proposed for the first time in this paper-may use new, inexpensive electronics to create systems better able to help workers take advantages of the benefits of procedures. Task guidance systems are defined by both the information they are- and are not—able to provide to the worker. All the information known a priori about the task can be preloaded into the task guidance system; procedures contain a wealth of information about the task in general — and how to complete it in particular-and are the source of information for a task guidance system that this article focuses on.

What task guidance systems do not know-and therefore cannot present to the worker-is information about the current state of the environment in general, and the operand (entity being inspected, maintained, or assembled by executing the procedure) in particular. The system does not contribute to a worker's situation awareness directly; its only source of current situation assessments is from the worker through his or her inputs. Of course, holding a system to the most rigorous definition of containing only task information may be extreme; for example, many electronic systems contain internal clocks, which, existential arguments aside, may be considered as providing environmental information. As such, task guidance systems may be classified as those task aids whose dominant purpose is providing preloaded task information, rather than current sensing about the environment at large.

By only requiring the task guidance system to provide task information, they themselves demand fairly simple technologies to implement. Recent research has focused on making the technology physically and economically viable for the specific characteristics of many tasks. For example,

1. Mobile workers need their aids to be light, easy to carry, and — if worn—comfortable. At the time of the writing of this article, personal data assistants fit into shirt pockets and wallets; the more capable wearable computers weigh only a couple of pounds and can be carried in a small waist or shoulder bag, with visual and auditory displays attached to eyeglasses or a headband.

2. Many workers-mobile or not-need at least one hand free to execute the procedure. One-handed keyboards, carried in the palm of the hand, can allow for quick data entry. Speech recognition is now capable of reliably interpreting discrete commands once calibrated to the worker, and is rapidly improving in capability and accuracy; its use is germane in both situations where the worker cannot touch input devices due to gloves or material on their hands such as oil or grease, and where he or she needs both hands free.

3. Some workers need to work from a variety of positions, such as a mechanic who may need to lean over or lie under an engine. In such situations, defined by tight spaces and unusual positions, small electronics can fit and be oriented to suit the worker, as compared to bulky manuals.

4. For their widespread use, the systems should be sufficiently inexpensive that distributing them to all workers on shop floors, for example, is possible and cost-effective. The recent mass-marketing of personal data assistants may be taken as an example of how this cost requirement is being met; although not as widely advertised, wearable computers are no longer singular products of research groups, but are also being commercially marketed.

The use of such simple technologies limits the capabilities of task guidance systems. Unlike more sophisticated automation, task guidance systems cannot perform elements of the 
procedure, cannot help the worker sense and process information about the environment, and cannot monitor for errors and anomalies. Because the human remains the effector of all actions and the primary concern is completing the task rather than using a task guidance system, this article has deliberately chosen to use the term worker.

However, the use of such simple technologies makes task guidance systems eminently suitable for a variety of tasks. Their use neither requires special design for each specific instantiation nor ties them down to a singular location. They do not need to be calibrated or physically reconfigured for the task. In other words, not only may they be physically mobile, but also they may be mobile between tasks by needing only a different software program started or a different "task database" accessed. The challenge in designing task guidance systems may be seen as "how to do the most benefit with as little technology as possible."

Task guidance systems have a substantially different interaction with the human than with intelligent systems such as automation or decision aids. As shown in Figure 1, operators of intelligent systems perform tasks through their systems. These systems sense operand and environment information, and provide a display of this information to the operator, often in a form where the information has been filtered and integrated; thus, the operator's perception of the environment is mediated by the systems. In some types of systems, such as decision aids, the system may also decide on courses of action; additionally, automation may also physically complete some elements of the task for the operator. In designing intelligent systems, many of the concerns revolve around the components of the task that the system can-and should - take over from the operator, and how the operator performs the task by operating this intermediate system.

In contrast, task guidance systems have no direct interaction with operand or environment information - and no ability to affect changes in the environment themselves. Instead, the worker performs the task directly, with the task guidance system having the orthogonal role of presenting predefined task information as a supplementary source of information and as a reminder.

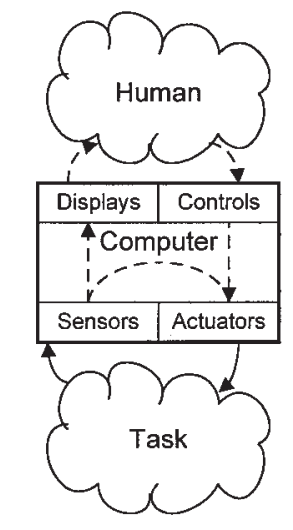

Interaction with Intelligent System

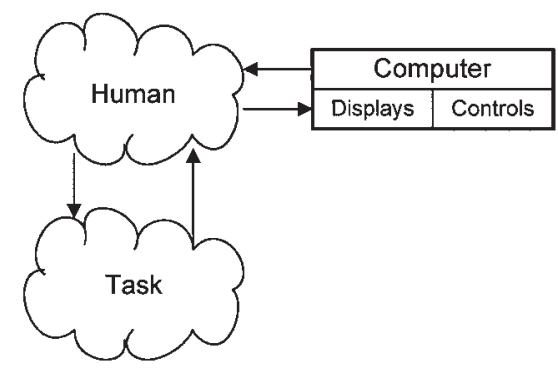

Interaction with Task Guidance

FIGURE 1 The human-task-computer relationship for intelligent systems and task guidance (based on diagram from Sheridan, 1992). 


\subsection{Design Guidelines for Task Guidance Systems}

Design guidelines for task guidance systems focus on two main goals: reinforcing the potential benefits of using a procedure for the task at hand; and mitigating any potential problems in using that procedure. These benefits and problems were discussed in the previous section-the following discussion relates them to the capabilities of task guidance systems.

3.3.1. Establishing task structure. Just as a procedure establishes a structure for the task, so may a task guidance system help communicate that structure to the worker. As the simplest mechanism, the task guidance system may present the steps to the worker as they are needed, mirroring the common application of written checklists. A more informative system may also provide a conceptual understanding of the task structure. This conceptual presentation could be global to the task-for example, a structural aircraft inspection task may indicate to the worker where he or she is relative to the aircraft, allowing them to see how far he or she has progressed. The presentation may also be local—for example, providing some anticipatory information about upcoming steps (Ockerman, Najjar, \& Thompson, 1998). Ordinality, irreversibility, and temporal considerations may also be described; for example, at each step, the subsequent steps that depend on this step may be noted, so that the worker is aware of the consequences of skipping each.

Providing information about task structure may be particularly useful when this structure is itself complex. For example, procedures may have hierarchical structures, in which high-level procedures refer the worker down to low-level detailed procedures; likewise, procedures may contain conditional statements at which point the procedure may fork in different directions. In such conditions, the task guidance system may be designed to allow the worker to review where in the task structure he or she is currently, and the steps that led there (Elm \& Woods, 1985).

3.3.2. Supporting memory. Like written procedures, task guidance systems can provide an external representation of a list of steps too long to remember. In addition, task guidance systems can also help workers keep track of their current location within the task. For example, if the system requires a cue from the worker when each step is concluded to know to move to the next step, then the system can provide an external, persistent memory of location within the task that is robust to memory lapses and interruptions. Likewise, task guidance systems may record when the worker elects to skip a step and later allow the worker to review these skipped steps when desired, or remind the worker later in the task that their current step depends on completing a previous step that was skipped and is not yet completed. Not only do preliminary research results suggest that such features help prevent known memory problems (Ockerman \& Pritchett, 1998), but also they may decrease the apparent need for a worker to follow the procedure's steps in the order written and thereby enable the worker to tailor their actions to immediate conditions.

3.3.3. Supporting consistency and safety. Not only may task guidance systems help workers complete a procedure thoroughly and reliably, as just described, but they may also then be given an easy mechanism to record that the task was completed-and how it 
was completed. Such records may then be downloaded to data-keeping applications, thereby providing detailed records without requiring data entry after the task (Ockerman, Najjar, \& Thompson, 1999). Such an institutional knowledge, resident at a common source of downloads, may also enable a more structured and reliable means of communication between team members. Completed steps, skipped steps, and pending steps can potentially be communicated between workers' task guidance systems, or uploaded from a central data record at the start of a shift.

3.3.4. Supporting worker expertise. Although workers may need considerable expertise, any worker can always learn more about a task if motivation exists and means are provided. Motivation aside, task guidance systems can easily allow the worker to access more detailed information, if the preprogramming investment of putting this information into the system is made (Ockerman, Najjar, \& Thompson, 1999). This information may be about the task at hand, such as describing to a mechanic how an engine part will move in operation to facilitate detecting if the part wear is out of range; likewise, this information may be about the procedure, such as describing the rationale for asking the mechanic to look at this engine component at this point in the procedure and how it fits into the higher level "general" procedure (Catrambone, 1990).

Once confident in their knowledge, the worker may then benefit from the ability to select less information about each step, reducing the time reading and processing task information. Allowing the deselection of information gives the individual worker control over the detail of their procedure, while keeping its underlying structure consistent between all workers.

3.3.5. Supporting effective task strategies. A procedure may serve as a lock-step sequence of directives or as a guideline, depending on the procedure's comprehensiveness, detail and accuracy, and on the worker's expertise. Beyond the just-mentioned self-calibration by the worker of the procedure's detail, task guidance systems may be able to indicate to the worker an effective and appropriate strategy, explicitly or implicitly. For example, a procedure step that serves as guidance may not only be worded as such (already advisable in developing written checklists), but also may allow the worker to view other possible actions, the rationale for each step, and other such supportive information. On the other hand, a step that absolutely must be performed as specified may be presented as a directive, perhaps with the accompaniment of an aural alert, and with the rationale given for performing the step exactly. The operator might not be allowed to check any subsequent steps as complete until the worker checks off the directive.

Likewise, task guidance may be able to encourage anticipatory strategies in procedure following. Possible mechanisms range from consistently providing the worker with a preview of upcoming steps, to identifying dependencies between steps that an anticipatory strategy can build on and using this knowledge, on a step-by-step basis, to show future steps that will be impacted by current actions.

3.3.6. Mitigating unintentional noncompliance. Although mitigating unintentional noncompliance may be an important goal in designing a task guidance system, 
achieving this goal may be more accurately viewed as a side effect of good system design. As such, reducing many of the manifestations of unintentional noncompliance-interruptions, losing track of current location in the procedure, forgetting which steps were skipped-have already been discussed in the preceding sections.

3.3.7. Mitigating intentional noncompliance. The most prevalent rationale given by workers for intentional noncompliance is that the procedure (or its physical instantiation) is too burdensome to be beneficial. Such conditions are named in studies of underreliance on automation, in which it has been demonstrated that automation that requires significant interaction and workload from its operator may not be used if the operator perceives he or she can complete the task more easily on his or her own (Kirlik, 1993). Unfortunately, if the underlying procedure is truly inefficient or ineffective, then this perception cannot be mitigated by a task guidance system, no matter how well designed.

When the procedure is a good foundation to develop a task guidance system on, design decisions can have a powerful impact on the burden perceived by the worker in using it. At its worst, the physical instantiation of a procedure may physically be burdensome, such as maintenance manuals comprising several thick volumes. Task guidance systems can potentially be quite light and small; specific standards for comfort can ultimately only be measured by workers on task for an entire shift. Likewise, poorly designed task guidance systems may impose a cognitive burden by such unforgivable characteristics as poor legibility, overly complex operation, and so forth.

To encourage an appropriate level of compliance, task guidance systems may be purposefully designed to help the operator understand the benefit of the procedure. For example, by presenting the overall intention of the procedure, the rationale for each step, and an illustration of the task structure, the worker may be able to develop a more accurate perception of the procedure's worth than if it is just presented as a series of steps to be taken on faith.

As another method of making its use less burdensome, a task guidance system may integrate other information activities into their associated steps. For example, paper maintenance manuals may frequently refer the mechanic to tables at the end of the book for specific parameters, such as desired engine oil viscosity as a function of ambient operating temperature, requiring the tables to be carried around separately or flipping between tables and checklist. Task guidance systems have the innate capability to present this information when and where it is needed, and to interactively present only the value that corresponds to specific inputs from the worker, rather than potentially large data structures. Likewise, many inspection tasks have an information gathering component, such as jotting down fluid levels, faults found, and maintenance to be done. Designing the task guidance system to not only prompt for the action but also then easily receive and record the result eliminates the need for a separate data-recording mechanism (Ockerman, Najjar, \& Thompson, 1999).

Intentional noncompliance may also arise when the worker perceives that the organization condones - or encourages - noncompliance. Investing in task guidance systems and encouraging their use can stress an organization's desire for all workers to follow procedures when that desire truly exists and is reflected in all aspects of management, pay and compensation, and the working environment.

Finally, workers may not comply with procedures when they fear the procedure is inaccurate. The ultimate goal of task guidance systems may be to enable the worker to recognize 
when a specific step is faulty or inappropriate for the situation by providing the worker with sufficient information about each step-its intention, the conditions under which it is assumed to be accurate, and so on-that he or she can judge its merits. Unfortunately, such a design goal may be difficult to achieve because the decision to follow a procedure can be very brittle. Once a fault has been perceived (whether the perception is right or wrong), the remainder of the procedure may be stereotyped as untrustworthy instead of each step being judged for its individual merits. This effect is similar to the effects of automation faults on operator trust and reliance (Lee \& Moray, 1994; Muir, 1987) and of false alarms on operator conformance to subsequent alerting system commands (Hasse, 1992; Pritchett, Vándor, \& Edwards, 1999).

3.3.8. Mitigating overreliance on the procedure. Just as workers may not trust a procedure once it has failed, so may they not mistrust it before a failure. This may lead to overreliance on the procedure, a condition where the worker effects a procedure that is "wrong." Analogies may be drawn from research on automation (reviewed in Parasuraman \& Riley, 1997), in which overreliance remains a sizeable concern. A procedure may be wrong in four ways. First, the procedure may be technically correct but have a different intention - and therefore different outcome - than the worker. Second, a procedure may be technically correct and have the desired intention, but may have been designed to work within a limited range of environmental boundary conditions that are currently not satisfied. It is reasonable to hypothesize that a task guidance system may help mitigate these two causes of overreliance by making the worker an educated follower of the procedure through providing sufficient information about the procedure to judge its appropriateness to the situation at hand.

The third potential problem with a procedure arises when the worker follows it using an inappropriate strategy. For example, an aircraft inspection task typically has an associated checklist, which asks the worker to inspect the most important aircraft components and the most likely sources of faults; however, knowing that no one procedure can foresee every possible problem with the aircraft, the inspector is expected to use the checklist as a guideline, while remaining observant for anything else that may appear out of order. For the inspector to execute this checklist exactly, yet miss observable faults, represents a form of overreliance on the procedure. Such overreliance may stem from carelessness, which a task guidance system cannot mitigate; or it may stem from a lack of awareness of the intended role of the procedure, which a task guidance system can help prevent through two nonexclusive approaches. First, the "warning label" approach may have the task guidance system explicitly remind the worker when any of the steps is only a guideline or point sample of a large set of tasks, reducing complacency and communicating expectations. Second, the cognitive-being approach attempts to situate the worker better in the task, so that the worker can see the layout of the suggested inspection tasks, know the upcoming tasks so that he or she can work toward them, and thereby actively use the task structure of the procedure as a basis for how he or she approaches the task.

Finally, the fourth potential manner in which a procedure may be wrong is when a step is erroneous; that is, in which the step does not meet its stated intention within its allowable boundary conditions. Like a mechanical fault in an automated system, this is a problem that should be tested for and prevented during the design of the procedure, as it requires substantial expertise and confidence to identify in operational conditions. 


\subsection{Task Guidance Studies}

Although task guidance is explicitly defined for the first time in this article, there are a number of studies that have been conducted that could be considered task guidance research. Each illustrates specific designs of task guidance systems and provides insights into the future direction of task guidance research. Many of these studies center on electronic procedures, that is, the electronic presentation of procedural steps.

An early study was done on the organization and presentation of procedures in an electronic format for the nuclear power domain (Elm \& Woods, 1985). An initial design of the procedures in an electronic format resulted in workers "getting lost" in the electronic procedures. For example, workers could not find the procedure that they wanted, could not skip easily from one procedure to another, and could not find steps they wanted to return to. After investigating how the paper procedures were used, the system was redesigned using an electronic book metaphor intended to support the worker in "looking ahead" in the current procedure while waiting for some action, to support the worker in returning to procedure steps left pending, to support worker monitoring of other related issues while in a procedure, and to support the worker in maintaining an awareness of the current context (i.e., how this step fits into the overall procedure). This allowed the worker to see the current procedure and the navigation methods simultaneously. There was also a "foldout" window that copied the function of foldout pages in the paper procedure to inform the worker of other items to be monitoring, a subroutine map, which showed the worker's previous path through the procedures, and a list of incomplete steps. Informal walkthroughs of the redesigned system indicated that getting lost was no longer a problem.

In a more recent study, electronic procedures were tried with airline maintenance personnel (Kesterson, Barker, Guay, \& Butler, 1998). The initial design, which used a book metaphor, was well accepted. However, some of the mechanics seemed to have trouble using it. It was found that knowledge of the maintenance process was a necessary prerequisite for performing well with the new aid as initially designed. Although the electronic book metaphor was applied to the design, observations showed that there were two methods of using the system: book-based and data-based. It was observed that the data method mimicked the method taught to mechanics, and the data-based workers were more proficient than the book-based workers. Therefore, the interface was redesigned to make the data method prominent and lead the mechanics through the troubleshooting and repair process in a manner consonant with their training. With the redesign, no training on the system was required and all the mechanics finished each test task in less time.

There has been substantial research done on the design of aircraft inspection procedures, with both paper and electronic formats. A series of studies demonstrated that paper procedures are more effective if guidelines on information readability, information content, information organization, and physical handling and environment are followed (Patel, Drury, \& Lofgren, 1994). When these guidelines were applied to electronic procedures, researchers found that the electronic procedures, which employed hypertext links, were rated much higher than the original paper procedures but only slightly higher than paper procedures developed within the same guidelines by commercial aircraft inspectors (Patel, Pearl, Koli, \& Drury, 1994). A recent investigation into electronic cockpit procedures for commercial airline pilots (Shamo, Dror, \& Degani, 1998) determined that the pilots had better performance with electronic procedures than the traditional paper-based procedures, as measured by speed and accuracy. In addition, the pilots liked the electronic procedures and found them easy to learn to use. 
In addition to electronic procedure experiments, there have been field studies of presenting procedures on wearable computers. Researchers at Carnegie Mellon University conducted two of these studies, looking at the feasibility of using wearable computers and focusing on the hardware reliability. One field study involved the inspection of military tanks. Having the wearable computer inspection system allowed the inspectors to go from a two-man workforce to one and also reduced inspection time (D. Siewiorek, personal communication, 1995). A second field study examined the use of electronic repair information for military jets (Siegel \& Bauer, 1997). The study compared the performance with the normal paper repair information to the performance of the electronic repair information on the wearable computer. Researchers found that the inspectors' performance improved because they followed the directions given on the wearable computer more closely; in this case, improvement was possible because the procedures were thorough and appropriate for the situation.

Another study examined worker performance at origami, the Japanese art of paper folding, when using a book compared to when using a head-mounted auditory and visual display with voice control (Ockerman, Thompson, \& Najjar, 1998). The study participants who used the book completed their origami project significantly faster than the participants using the computer did, although there was no statistical difference in the accuracy of the two groups. The participants using the book had an advantage; all of their instructions were contained on one page of the book. The book users were observed looking at the future steps and used this information to form intermediate goals that helped them understand the purpose of the current step. In comparison, the computer users had to go to a different screen for each step, requiring more time and preventing the computer users from knowing what the next step would entail; in other words, the computer users had no easy way to look ahead. In origami this is particularly useful; for example, the one computer user who had previously done origami would go to the next step to see what he should end up with. This study emphasized the importance in some tasks of being able to look ahead for intermediate goals or for planning what to do next, and illustrated how task guidance systems that only present the current step may create difficulty for the user, findings also reported in a study of nuclear power plant computerized procedures (Jeffroy \& Charron, 1997).

Another investigation of a task guidance system looked at preflight inspection of general aviation aircraft (Ockerman \& Pritchett, 1998). In this study, one third of the participants did the preflight inspection by memory (which is common among general aviation pilots) and the other two thirds used electronic preflight checklists on a wearable-computer task guidance system. The preflight inspection checklist given by the task guidance system, while identical to the one provided by the manufacturer of the plane, is not very detailed and did not prompt the pilots to check all the features of the aircraft. Due to the small number of participants, there were few statistical differences. However, there was some evidence that the pilots were overrelying on the task guidance system to tell them what to do, as the pilots using the wearable-computer system did few, if any, checks beyond those in the checklist. In the debriefing questionnaire 9 of the 10 pilots who used the task guidance system reported doing the preflight inspection differently from normal, with comments such as "It was easier to follow the system than to think about what normally is done."

\subsection{Studies of Related Automation}

Automatic checklists build on the capabilities of electronic checklists, but so do all the items that it has physical control over. Although their apparent function is very similar to task guid- 
ance systems, from the worker's point of view, automatic checklists necessarily require a significantly larger investment to implement, due to their need for sensing and actuation capabilities, and are normally specialized for and fixed to a particular operand. Automatic checklists are also necessarily limited to procedures with a high level of detail because the procedures must be able to exactly specify what actions to take in executing each step.

Tests of automatic checklists have examined several different levels of automation. For example, an automatic checklist for cockpits might include a step examining an engine parameter; a checklist with a lower level of automation might provide a recommendation to the pilot that the engine can be checked-off as OK, and a checklist with a higher level of automation might check-off steps transparently to the pilot. Although this design of automatic procedures was done to improve safety by providing a redundant method of completing procedures, the crew would tend to depend on the automation and not make their own checks, missing critical faults; the more automated the system, the more likely the crew would miss important items (Mosier, Palmer, \& Degani, 1992; Palmer \& Degani, 1991). Similar concerns about the potential for overreliance and the role of the worker were raised in a study of automatic and electronic checklists in nuclear power plants (Spurgin, Wachtel, \& Moieni, 1993).

Similar to automatic systems intended to help workers complete procedures are automatic systems intended to help workers with "task management," the prioritization, initiation, monitoring, and completion of multiple tasks that are concurrently vying for the operator's attention (Funk, 1991; Rogers \& Mosier, 1996). Unlike task guidance, task management aids have knowledge of what the worker has done, the environment, the operand, and any instructions that have been given to the worker. Task management aids have been tested as cockpit systems, where the system informs the pilot of tasks to be completed and displays a color-coded list of the tasks that are imminent (would need attention in the near future), in process and proceeding satisfactorily, in process and proceeding unsatisfactorily, or late in being initiated. With the use of a low-fidelity cockpit simulator, it was shown that pilots had better task management performance, as measured by response time, percentage of misprioritization, percentage of unsatisfactory controls, and percentage of incomplete tasks, when they used a task support system (Funk, 1991; Funk \& Kim, 1995). The pilots also liked the task management aid used in the experiment. However, there are several caveats to this success. As is described in Funk (1991), the cost, weight, and space needs of the sensors required by an intelligent task management aid are much beyond what current and even foreseeable future planes will be able to carry and afford. In addition, there is no guarantee that the sensors currently available or available in the near future will be accurate enough to support a comprehensive task management aid; instead it may only have knowledge of some fraction of the tasks, making accurate task prioritization difficult. In conditions where the accuracy of the sensors is a problem, the pilots will be required to monitor another system. Finally, it has been shown in some cases that human operators do not like to be told what to do by a computerized system on a minute-by-minute basis (Knaeuper \& Morris, 1984).

\section{PROCEDURE CONTEXT}

Throughout the discussions to this point in the article, specific information has been repeatedly noted that task guidance systems should provide, both in first-principles discussions of providing aid to workers and in summaries of experimental studies. These information elements are all based on the principle that, without sufficient information to make decisions 
and to assess the situation, the worker is reduced to following the instructions one by one (Swezey, 1987). This procedure context information enables the worker to use the procedure to his or her benefit and judge its accuracy and merit, by situating each step in the larger purpose and structure of the procedure.

Several specific pieces of procedural context information have been specifically discussed in this paper, including

1. Intention of the overall procedure.

2. Rationale for each individual step.

3. Boundary conditions within which the procedure (or individual step) is assumed to be accurate.

4. Appropriate specificity of the procedure or its individual steps or both, indicating whether it is considered to be sufficiently detailed or it is to be used as a guideline.

5. Forking of procedure due to assessments of environmental conditions.

6. Location information within the task, such as indicating where the user currently is in the task in terms of time, number of steps, or position.

7. Previous actions, providing detail about the immediately past procedural steps.

8. Following actions, providing detail about soon-to-occur procedural steps.

9. Ordinality, indicating the order of the steps and perhaps an explanation for the choice of this order.

10. Necessity, indicating whether each step must be carried out, or is optional, or conditional on specific environmental factors.

11. Reversibility of a step.

12. Triggering conditions that must be present before a task is started.

13. Temporal construct information, such as the period of time in which an action can or should be performed.

Some of these information elements are typically implicit in written checklists, such as location within the task, previous actions, and following actions. Explicitly providing this information on task guidance systems is motivated by several concerns. First and foremost, experimental prototype task guidance systems to date have initially just focused on presenting the worker with isolated steps, and have fairly consistently then reported the need for this information, especially when the procedure was being used as a guideline and anticipatory strategies were appropriate (Elm \& Woods, 1985; Ockerman \& Pritchett, 1998; Ockerman, Najjar, \& Thompson, 1997; Siegel \& Bauer, 1997). Second, many systems suitable for task guidance have small visual displays and/or aural displays, both of which can present limited information; therefore, the worker will not typically be able to view at one time the entire procedure the same way it can be experienced with a paper checklist and will have less access to this information, unless vital elements are identified and explicitly provided.

The other procedure context elements are not typically included in written checklists in detailed form, yet may have utility to the worker. Many of these elements explain the procedure-its intentions, its capabilities, its underlying structure-and are typically known to the procedure designer, but then not necessarily communicated to the worker. As such, the presentation of this information by the task guidance system will require procedure designers to provide more substantive documentation of their design thought processes, knowledge that task guidance systems may then constructively present to the worker. 


\section{SUMMARY AND CONCLUSIONS}

Procedures are so useful that they are required in many types of tasks, such as inspection, assembly, and maintenance. They provide an effective, tested, and proven structure by which to complete the task, coordinate between workers, and help ensure consistency and safety. Task guidance systems can build on these inherent strengths, while they also help eliminate the problems that commonly occur with written procedures.

Task guidance systems have the potential to be much more than an electronic instantiation of a written list of steps. Task guidance systems can inherently be configured and programmed to meet the worker's needs. For example, the worker may be given control over the amount of detail to be shown, allowing each worker to tailor the task aid to his or her level of knowledge while ensuring that each worker uses a consistent task structure. Likewise, task guidance systems can be given awareness of interdependencies between steps in the procedure, giving the worker more flexibility by allowing him or her to do the steps in a preferred order while providing the safety mechanism of identifying any steps that need to be completed before the one currently presented.

However, many issues warrant further study. Although this article identified information that may be particularly helpful to present to workers, many task guidance systems may not have suitably large display space to present it all simultaneously, requiring careful insight, during design, of what information should be presented directly, what information is accessible, and how the worker might access it. Preliminary studies of task guidance systems have also identified the potential for workers to get lost in the system, requiring presentation of not just the immediate step, but also information about the context of the procedure.

With the presentation of information about the context for which the procedure is appropriate, task guidance systems can potentially give the worker an insight into the procedure designer's thought processes and assumptions. Not only does the information have the potential to mitigate glaring problems with inappropriate under and overreliance on procedures, but it also may help in more subtle ways, such as helping implicitly train the worker on the task structure, improving worker acceptance of and agreement with the procedure, and allowing the worker to knowledgeably improve on the procedure.

Throughout this article, we have maintained the position that procedures are important-sometimes vital - to successfully completing a complex task. Accurate, useful procedures may not be detailed, they may not be comprehensive-they may not even be appropriate for all situations - but they do provide a framework that the worker can build on as his or her expertise allows. The mention of procedures normally brings to mind the prescribed set of actions dictated by management and instantiated by written checklists, but this reveals only a narrow subset of the procedures that may be provided to a worker; a more inclusive view also accounts for guidelines and for the to-do lists that workers, independent of outside experts and management, informally arrange for themselves and pass on to "the new guy."

In instantiating a procedure into a hardware form - a task guidance system - it is imperative that the correct procedure is selected. When using paper checklists, workers may commonly use their own procedures, with or without the knowledge of the management; when presented with a task guidance system, on the other hand, they can no longer cut, delete, and modify the given procedure into the appropriate procedure with the same ease. Because task guidance systems formalize procedure to a greater extent than ever before, care must be 
taken to ensure that they are providing a correct, viable procedure that truly supports the worker.

In some domains, the correct, viable procedure is well known, and the worker is expected to follow it. Unfortunately, in other domains procedures are too commonly found to be faulty, inappropriate, or out of date. Just as machinery may be designed wrong or let to breakdown, so may procedures be designed incorrectly or not changed to meet new circumstances. Unfortunately, failures in machinery are easily visible and usually fixed as part of good business practices; procedures, on the other hand, are not always recognized institutionally as entities requiring design, testing, and maintenance. Their failures are not always manifested in the worker's behavior because he or she is able - through undue exertion - to compensate for their failures and, in some situations, to unofficially write their own procedures. The implementation of task guidance systems makes interaction with prescribed procedures more compelling to the worker, lowering their ability to compensate for faulty instructions and increasing the need for the organization to provide the worker with a correct procedure just as the correct mechanical tools are provided.

As such, the implementation of task guidance systems places requirements on the organization in which they will be used. The benefits of procedures can only be built on with task guidance systems when the procedures do not give erroneous instructions; the procedures do not necessarily need to be detailed, nor do they need to be comprehensive, but they do need to meet their promises to the worker within their stated capabilities. Organizationally, procedures will need to be accepted by the workers, and their use fully encouraged by management.

\section{REFERENCES}

Catrambone, R. (1990). Specific versus general procedures in instructions. Human Computer Interaction, 5, 49-93. Cessna Aircraft Company. (1975). Pilots operating handbook: Model 172 and Skyhawk. Wichita KS: Author.

Chen, T. L., \& Pritchett, A. R. (2000, January). On-the-fly procedure development for flight re-planning following system failures. The 38th AIAA Aerospace Sciences meeting and exhibit, Reno, NV.

Covault, C. (1999, August 23). Plug wrap, procedures led to IUS failure. Aviation Week and Space Technology, 34.

Degani, A., \& Wiener, E. L. (1994). On the design of flight-deck procedures (NASA Contractor Report 177642). Moffett Field, CA: NASA Ames Research Center.

Degani, A., \& Wiener, E. L. (1997). Procedures in complex systems: The airline cockpit. IEEE Transactions on Systems, Man, and Cybernetics-Part A: Systems and humans, 27, 302-312.

Drury, C. G., \& Prabhu, P. (1996). Information requirements of aircraft inspection: Framework and analysis. International Journal of Human-Computer Studies, 45, 679-695.

Drury, C. G., \& Sarac, A. (1997). A design aid for improved documentation in aircraft maintenance: A precursor to training. Paper presented at the 41st annual meeting of the Human Factors and Ergonomics Society, Santa Monica, CA.

Elm, W. C., \& Woods, D. D. (1985). Getting lost: A case study in interface design. Paper presented at the 29th annual meeting of the Human Factors Society, Santa Monica, CA.

Funk, K. (1991). Cockpit task management: Preliminary definitions, normative theory, error taxonomy, and design recommendations. The International Journal of Aviation Psychology, 1, 271-285.

Funk, K., \& Kim, J. N. (1995). Agent-based aids to facilitate cockpit task management. Paper presented at the IEEE Conference on Systems, Man, and Cybernetics.

Goodman, P. C., \& DiPalo, C. A. (1991). Human factors information system: A tool to assess error related to human performance in US nuclear power plants. Paper presented at the 35 th annual meeting of the Human Factors and Ergonomics Society.

Grosjean, V. (1995). Temporal strategies of operators feeling with numerous parallel processes: A field study. International Journal of Human Factors in Manufacturing, 5, 123-137. 
Hasse, D. (1992, November). ALPA ground proximity warning system survey. Proceedings of the Flight Safety Foundation 45th annual International Air Safety Seminar, Long Beach, CA.

Hutchins, E. (1995). Cognition in the wild. Cambridge, MA: MIT Press.

Institute of Nuclear Power Operations. (1986). A maintenance analysis of safety significant events. Atlanta, GA: Institute of Nuclear Power Operations (INPO).

Jeffroy, F., \& Charron, S. (1997). From safety assessment to research in the domain of human factors: The case of operations with computerised procedures. Paper presented at IEEE 6th annual Human Factors Meeting, Orlando FL.

Kershner, W. K. (1976). The advanced pilot's flight manual (4th ed.). Ames: Iowa State University Press.

Kesterson, B., Barker, R., Guay, R., \& Butler, K. (1998, May). Designing a portable maintenance aid (PMA) for user centered requirements. Paper presented at the 1998 International Conference on Human-Computer Interaction in Aeronautics, Montreal, Canada.

Kirlik, A. (1993). Modeling strategic behavior in human-automation interaction: Why an "Aid" can (and should) go unused. Human Factors, 35, 221-242.

Knaeuper, A., \& Morris, N. M. (1984). A model-based approach for online aiding and training in process control. Paper presented at the 1984 IEEE International Conference on Systems, Man, and Cybernetics, Halifax, Nova Scotia.

Latorella, K. A. (1996). Investigating interruptions: An example from the flight deck. Paper presented at the 40th annual meeting of the Human Factors and Ergonomics Society.

Lee, J. D., \& Moray, N. (1994). Trust, self-confidence and operators' adaptation to automation. International Journal of Human-Computer Studies, 40, 153-184.

Linde, C., \& Goguen, J. (1987). Checklist interruption and resumption: A linguistic study (NASA-CR-177460). Washington, DC: The National Aviation and Space Administration.

Marsden, P. (1996). Procedures in the nuclear industry. In N. Stanton (Ed.), Human factors in nuclear safety (pp. 99-116). London: Taylor \& Francis.

McCarthy, J. C., Wright, P. C., Monk, A. F., \& Watts, L. A. (1998). Concerns at work: Designing useful procedures. Human-Computer Interaction, 13, 433-457.

Mosier, K. L., Palmer, E. A., \& Degani, A. (1992). Electronic checklists: Implications for decision making. Paper presented at the 36th annual meeting of the Human Factors Society, Santa Monica, CA.

Muir, B. M. (1987). Trust between humans and machines, and the design of decision aids. International Journal of Man-Machine Studies, 27, 527-539.

Ockerman, J. J., \& Pritchett, A. R. (1998, May). Preliminary study of wearable computers for aircraft inspection. Proceedings of the International Conference on Human-Computer Interaction in Aeronautics, Montréal, Canada.

Ockerman, J. J., Najjar, L. J., \& Thompson, J. C. (1997). Wearable computers for performance support: Initial feasibility study. Personal technology, 1, 251-259.

Ockerman, J. J., Thompson, J. C., \& Najjar, L. J. (1999). FAST: Future technology for today's industry. Computer Industry, 38, 53-64.

Palmer, E., \& Degani, A. (1991). Electronic checklists: Evaluation of two levels of automation. Paper presented at the sixth International Symposium on Aviation Psychology, Columbus, OH.

Parasuraman, R., \& Riley, V. (1997). Humans and automation: Use, misuse, disuse, abuse. Human Factors, 39(2), 230-253.

Patel, S., Drury, C. G., \& Lofgren, J. (1994). Design of workcards for aircraft inspection. Applied Ergonomics, 25, 283-293.

Patel, S. C., Pearl, A., Koli, S., \& Drury, C. G. (1994). Design of portable computer-based workcards for aircraft inspection (Department of Transportation, Federal Aviation Administration DOT/FAA/AM-94/xx). Buffalo: Department of Industrial Engineering, State University of New York at Buffalo.

Pearl, A., \& Drury, C. G. (1995). Improving the reliability of maintenance checklists (DOT/FAA/AM-95/Xx). Washington, DC: Federal Aviation Administration, Biomedical and Behavior Sciences Division, Office of Aviation Medicine, Department of Transportation.

Pritchett, A. R., Vándor, B., \& Edwards, K. (1999, June). Testing and implementing cockpit alerting systems. Presented at the International Workshop on Human Error, Safety, and System Design, Liege, Belgium.

Rogers, W., \& Mosier, K. (1996). Understanding flight deck task management: A prelude to human-centered design. Paper presented at the 40th annual meeting of the Human Factors and Ergonomics Society, Santa Monica, CA. 
Roth, E. M. (1997). Analysis of decision making in nuclear power plant emergencies: An investigation of aided decision making. In C. E. Zsambok \& G. Klein (Eds.), Naturalistic decision making (pp. 175-182). Mahwah, NJ: Lawrence Erlbaum Associates, Inc.

Schutte, P. C., \& Trujillo, A. C. (1996). Flight crew task management in non-normal situations. Paper presented at the 40th annual meeting of the Human Factors and Ergonomics Society, Santa Monica, CA.

Seminara, J. L., Gonzalez, W. R., \& Parsons, S. G. (1976). Human factors review of nuclear power plant control room design (Electric Power Research Institute Report NP-309, Chapter 17).

Shamo, M. K., Dror, R., \& Degani, A. (1998). Evaluation of A new cockpit device: The integrated electronic information system. Paper presented at the 42nd annual meeting of the Human Factors and Ergonomics Society, Chicago.

Sheehan, J. J., \& Drury, C. G. (1971). The analysis of industrial inspection. Applied Ergonomics, 2, 74-78.

Sheridan, T. B. (1992). Telerobotics, automation and human supervisory control. Cambridge MA: MIT Press.

Siegel, J., \& Bauer, M. (1997, October). A field usability evaluation of a wearable system. Paper presented at the first International Symposium on Wearable Computers, Cambridge, MA.

Spurgin, A. J., Wachtel, J., \& Moieni, P. (1993). The state of practice of computerized operating procedures in the commercial nuclear power industry. Paper presented at the 37th annual meeting of the Human Factors and Ergonomics Society, Santa Monica, CA.

Swezey, R. W. (1987). Design of job aids and procedure writing. In G. Salvendy (Ed.), Handbook of human factors (pp. 1039-1057). New York: Wiley.

Thatcher, V. S. (Ed.). (1980). The new Webster encyclopedic dictionary of the English language. Chicago: Consolidated.

Thomas, D. L. (1995). Integrated maintenance information system (IMIS): User field demonstration and test (Technical Report AL/TR-1995-0034). Dayton, OH: Armstrong Laboratories, Wright-Patterson Air Force Base.

Vicente, K. J. (1999). Cognitive work analysis. Mahwah, NJ: Lawrence Erlbaum Associates, Inc.

Xiao, Y., Milgram, P., \& Doyle, D. J. (1997). Planning behavior and its functional role in interactions with complex systems. IEEE Transactions on Systems, Nan, and Cybernetics-Part A: Systems and humans, 27, 313-324.

Zach, S. E. (1980). Control room operating procedures: Content and format. Paper presented at the 41st annual meeting of the Human Factors and Ergonomics Society, Santa Monica, CA. 